\title{
积云对流参数化方案对热带大气季节内振荡 模拟的影响
}

\author{
胡文婷 ${ }^{(12)}$ ，段安民 ${ }^{(1 *}$ ，吴国雄 ${ }^{11}$ \\ (1) 中国科学院大气物理研究所大气科学和地球流体力学数值模拟国家重点实验室, 北京 100029 ; \\ (2) 中国科学院研究生院, 北京 100049 \\ * 联系人, E-mail: amduan@mail.iap.ac.cn
}

收稿日期: 2010-06-03; 接受日期: 2010-10-12

国家重点基础研究发展计划(编号: 2010CB951703, 2009CB421403)、中国科学院知识创新项目(编号: KZCX2-YW-Q11-01, KZCX2-YWBR-14)和中国科学院战略性先导科技专项(编号: XDA05110303)资助

\begin{abstract}
摘要本文利用中国科学院大气物理研究所大气科学和地球流体力学数值模拟国家 重点实验室(LASG)的大气环流谱模式的最新版本 SAMIL2.2.3 系统分析了积云对流参数 化方案对热带大气季节内振荡(ISO)模拟的影响. 结果表明该模式的三种积云对流参数 化方案都能模拟出热带地区气候平均季节内振荡(CISO) 时空演变的基本特征; 其中 Tiedtke 方案相比于 Manabe 方案和 Zhang-McFarlane 方案, 模拟出的 CISO 与观测更为接 近. 而 SAMIL2.2.3 模式对 ISO 年际变化部分(TISO)的模拟结果显示尽管 SAMIL2.2.3 相 对于之前的版本对 Tiedtke 方案和 Zhang-McFarlane 方案针对不同问题做了必要调整, 但 三种积云参数化方案中只有 Zhang-McFarlane 方案能够模拟出 27 50 天 TISO 模态的东 传特征. 另外, SAMIL2.2.3 与其较早版本 SAMIL2.08 中的 Manabe 方案完全相同, 物理 过程的其他部分如辐射方案和气溶胶条件有所调整, 但仅后者能模拟出 $100^{\circ} \mathrm{E}$ 以西热带 27 50 天 TISO 模态的东传特征. 因此, 模式发展过程中物理参数化方案的改进并非在每 一方面上都产生正面效果。动力框架和物理过程, 以及不同物理过程之间的协调发展是 改进模式的重要途径.
\end{abstract}

关键词

积云对流参数化方案

季节内振荡

大气环流模式

非绝热加热廓线
热带地区季节内振荡(Intraseasonal Oscillation, 简称 ISO) 是大气中最显著信号之一, 也是影响全球 天气和气候的重要现象. Madden 和 Julian ${ }^{[1 \sim 3]}$ 利用 Canton 岛气压和西风分量的多年资料, 分析得到非 常显著的 41 53 d 周期的季节内振荡, 之后又扩展到 全球的热带海洋. 随后各国气象学家对 ISO 的研究一 直在进行, 发现热带大气不但有 30 60 d 准周期振
荡 $^{[4 \sim 6]}$, 还有 10 20 d 准双周振荡 ${ }^{[7,8]}$. 各种研究表明 热带 ISO 对亚洲夏季风的爆发与撤退 ${ }^{[9]}$, 热带风暴活 动 ${ }^{[10]}$ 和 ENSO 现象 ${ }^{[11,12]}$ 都有一定程度的影响, 是从 短期天气变化到长期天气变化中间的重要一环. 研 究热带 ISO 的目的不仅仅是描述其特征和规律, 更重 要的是, 它还为提高动力季节预测水平提供理论依 据.

英文引用格式: Hu W T, Duan A M, Wu G X. Sensitivity of simulated tropical intraseasonal oscillations to cumulus schemes. Sci China Earth Sci, 2011, doi: 10.1007/s11430-011-4215-0 
模式对 ISO 的预报误差会影响天气预报结果的 可信度水平, 不论是三天还是十几天的预报 ${ }^{[13]}$. 目前 大气环流模式对 ISO 的模拟能力还不高. 在国际大气 环流模式比较计划(AMIP)中, Slingo 等 ${ }^{[14]}$ 比较 15 个 大气环流模式模拟大气 ISO 的结果, 发现虽然大多数 模式能够反映大气季节内时间尺度的振荡信号, 但 严格来说，没有一个模式能够抓住观测到的热带 ISO 的主要特征.

由于不同模式所用的动力框架和物理过程不尽 相同, 因而影响大气环流模式模拟热带 ISO 的因子也 有所不同, 主要可以归结为三点: 模式分辨率、海气 相互作用及其积云对流参数化方案. 贾小龙和李崇 银 ${ }^{[15]}$ 利用 SAMIL-R42L9 研究了热带大气季节内振荡 (MJO) 的模拟对积云对流参数化方案的敏感性, 结果 表明两种对流参数化方案 (Manabe 方案和 Zhang-McFarlane 方案) 对 MJO 的模拟能力有明显的 差异. 除此之外, 更多的研究 ${ }^{[16 ~ 18]}$ 表明: 若大气环流 模式采用不同的积云对流参数化方案, 其结果是有 很显著差别的, 说明对流参数化方案对模拟热带 ISO 起着非常关键的作用.

Wang 等 ${ }^{[19]}$ 发现北半球夏季季风区存在显著的 气候态季节内振荡(CISO), 它代表 ISO 中锁相于年循 环的部分, 并且指出 CISO 的极端位相对应着夏季风 的演变特征. 而 $\mathrm{TISO}^{[20]}$ 是总的 ISO 当中除去 CISO 分量之后剩下的那部分, 代表着 ISO 年际变化的成分, 该部分对季节内尺度极端事件的发生有密切的关系. 杨静 ${ }^{[20]}$ 分析了中国科学院大气物理研究所大气环流 格点模式 GAMIL1.1.1 对夏半年东亚-西北太平洋地 区 CISO 和 TISO 的模拟能力, 发现尽管 GAMIL1.1.1 能够模拟出 CISO 以及 TISO 的一些主要特征, 但却 不能重现向东传播的 27 50 d 振荡信号.

基于以往 ISO 的数值模拟研究, 并鉴于 CISO 与 TISO 的实际意义, 本文将系统分析一个大气环流模 式 SAMIL2.2.3 对 CISO 和 TISO 的模拟水平. 通过比 较不同的对流参数化方案的结果, 研究积云对流参 数化方案对模拟 ISO 的影响, 并探讨其可能原因.

\section{1 资料、模式介绍及分析方法}

\section{1 资料}

（1）美国海洋和大气局(NOAA)1980 2006 年日 平均大气顶向外长波辐射 (Outgoing Longwave
Radiation, OLR) ${ }^{[21]}$.

(2) NCEP/DOE1980 2006 年再分析资料集 II $^{[22]}$.

\section{2 模式介绍}

本文所采用的大气环流模式是中国科学院大气 物理研究所大气科学和地球流体力学数值模拟国家 重点实验室所发展的高分辨率全球大气环流谱模式, 简称 SAMIL(Spectral Atmospheric Model of IAP LASG). 水平分辨率为菱形 42 波截断, 相当于经纬 网格 $2.8125^{\circ}$ (经度) $\times 1.67^{\circ}$ (纬度), 并形成了配套的外 强迫数据; 垂直分辨率取 26 层. 在动力框架方面, 采 用了参考大气扣除的方法 ${ }^{[23]}$, 并且引入了半隐式时 间积分方法. 辐射方案来自 Edwards 和 Slingo ${ }^{[24]}$, 而 陆面过程为 $\mathrm{SSiB}$ 模式 ${ }^{[25,26]}$. 模式的其他具体介绍可 参考文献[27].

本文采用的是 SAMIL 的最新版本 SAMIL2.2.3, 它共有三种积云对流参数化方案可供选择, 分别为 Manabe 方案 ${ }^{[28]} 、$ Zhang-McFarlane 方案和 Tiedtke 方 案, 其中 Zhang-McFarlane 方案 ${ }^{[29,30]}$ 与 Tiedtke 方案 ${ }^{[31]}$ 有一定程度的改进.

\section{3 试验设计和分析方法}

分别采取 SMAIL 2.2.3 模式中三种积云对流参 数化方案进行三组模拟实验. 模式运行采用国际大 气环流模式比较计划 AMIP II (http://www-pcmdi. 1lnl.gov/projects/amip/AMIP2EXPDSN/BCS_OBS/ami p2_bcs.htm)20 年平均的海温海冰场, 初始场采用 1979 年 1 月 1 日 NCEP/DOE 再分析资料. 每组试验 积分 28 年, 取后 27 年作为分析数据. 为便于比较, 观测资料(日平均 OLR 和 NCEP/DOE 再分析资料)选 取为 1980 2006 年, 用 CISO 和 TISO 两个指标来评 估在不同的积云参数化方案下 ISO 的模拟性能.

利用傅里叶分析把气候态场 $y_{\mathrm{c}}(i), i=1,365$ 分为 三个部分: $y_{\mathrm{ciso}}(i)=y_{\mathrm{c}}(i)-y_{\mathrm{ac}}(i)-R(i)$, 其中 $y_{\mathrm{ac}}$ 代表前三 个傅里叶谐波之和, 即年循环; $R$ 代表天气尺度扰动 (用 $5 \mathrm{~d}$ 滑动平均去除). CISO 即为气候态场去掉年循 环, 再用 $5 \mathrm{~d}$ 滑动平均除去噪音后得到. TISO 是从原 始日时间序列, 通过除去气候态, 再用 $5 \mathrm{~d}$ 滑动平均 滤掉天气尺度扰动后得到. 本文中所讨论的 CISO 和 TISO, 观测分析是由 OLR 日平均资料进行计算的, 而模式分析是由模式模拟的日降水量进行计算的.

本文主要研究亚洲三个典型热带季风区域的 
ISO 特征, 即热带印度洋地区 $\left(10^{\circ} \sim 20^{\circ} \mathrm{N}, 50^{\circ} \sim 75^{\circ} \mathrm{E}\right)$, 孟加拉湾地区 $\left(10^{\circ} \sim 20^{\circ} \mathrm{N}, 80^{\circ} \sim 100^{\circ} \mathrm{E}\right)$ 和南海地区 $\left(10^{\circ} \sim 20^{\circ} \mathrm{N}, 110^{\circ} \sim 120^{\circ} \mathrm{E}\right)$. 为简便起见, 以下章节均 用 IDO, BOB 与 SCS 表示. 本文的主要统计诊断方法 包括单点超前滞后相关分析, 带通滤波, 傅里叶谐波 分析等.

\section{SAMIL2.2.3 模式对 CISO 的模拟}

Yasunari $^{[32]}$ 在 1979 年第一次发现在南亚季风区 从赤道到 $30^{\circ} \mathrm{N}$ 存在与 ISO 相关的明显的云和积云对 流向北传播. 这种阶段性的北传特征可以很好地通 过 CISO 来体现. 下面分别利用三种积云对流参数化 方案的模拟结果, 对三个区域(BOB, IDO 和 SCS)经 度带进行平均, 比较分析三个区域 CISO 的经向传播
特征. 从 CISO 着手评估三种积云对流参数化方案对 模式模拟能力的影响.

图 1(a)表明观测中的北半球夏季 $\operatorname{IDO}\left(50^{\circ} \sim 75^{\circ} \mathrm{E}\right)$ 平均的 CISO 经向传播特征, 该区域 CISO 的信号可 从赤道向北传播至 $30^{\circ} \mathrm{N}$. Manabe 方案(图 1(d))和 Zhang-McFarlane 方案(图 $1(\mathrm{~g})$ )模拟结果显示 $10^{\circ} \mathrm{N}$ 以 北 CISO 信号几乎消失, 在 $10^{\circ} \mathrm{N}$ 以南 Manabe 方案中 的 CISO 信号比 Zhang-McFarlane 方案中的要弱. 只 有在 Tiedtke 方案(图 1(j))模拟结果中 CISO 信号延伸 到了 $20^{\circ} \mathrm{N}$, 并且干湿位相非常清楚.

观测(图 1(b)) 显示北半球夏季 $\mathrm{BOB}\left(80^{\circ} \sim 100^{\circ} \mathrm{E}\right.$ ) 平均的 CISO 湿位相及其前后的两个干位相十分规律 地向北传播，湿位相爆发发生在五月中旬，其北传速 度平均每天 0.4 个纬距. 与观测结果相比, Manabe 方 案(图 1(e))和 Tiedtke 方案(图 1(k)) 可以大致地模拟出
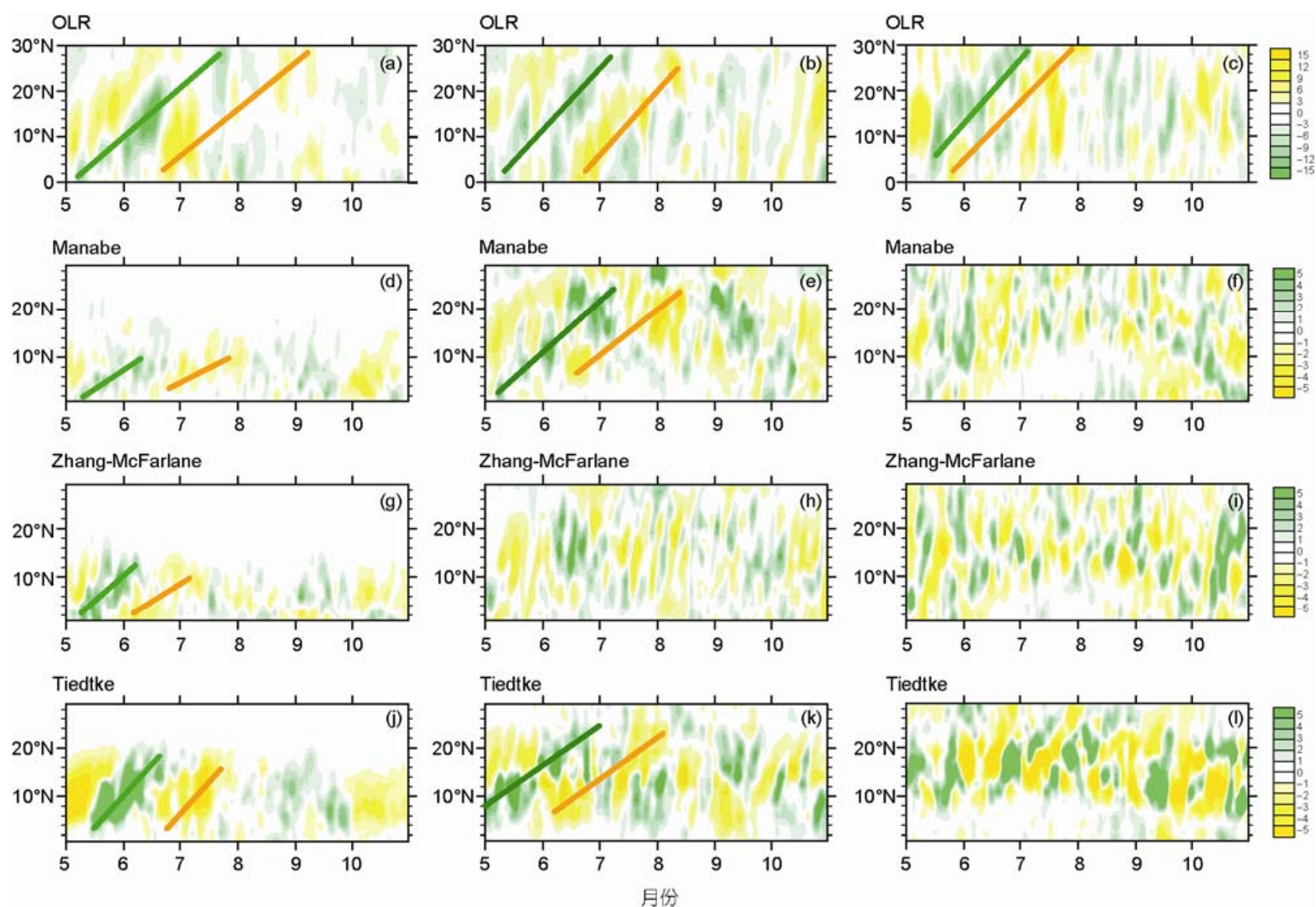

图 1 北半球夏季经度平均的 CISO 经向传播特征

图中第一列为 $\operatorname{IDO}\left(50^{\circ} \sim 75^{\circ} \mathrm{E}\right)$ 平均, 第二列为 $\mathrm{BOB}\left(80^{\circ} \sim 100^{\circ} \mathrm{E}\right)$ 平均, 第三列为 $\operatorname{SCS}\left(100^{\circ} \sim 120^{\circ} \mathrm{E}\right)$ 平均. 所用资料: 第一行 $\mathrm{OLR}$ 观测资料; 第 二行为 Manabe 方案所得模拟日降水量; 第三行为 Zhang-McFarlane 方案所得模拟日降水量; 第四行为 Tiedtke 方案所得模拟日降水量. 黄色 区域代表降水偏少(干异常)，绿色区域代表降水偏多(湿异常)。单位: $\mathrm{W} \mathrm{m}^{-2}$ (OLR), $\mathrm{mm} \mathrm{day}^{-1}$ (降水) 
CISO 的主要特征, 仍然存在缺陷, 例如就湿位相爆 发时间与北传速度而言, Manabe 方案与 Tiedtke 方案 模拟爆发时间偏早且北移速度偏慢, Manabe 方案模 拟速度为每天 0.36 个纬距, Tiedtke 方案模拟速度为 每天 0.3 个纬距; 与之相应的湿位相后的干位相出现 日期也偏早而其传播速度也偏慢. 而 ZhangMcFarlane 方案(图 1(h)) 没有成功模拟出 CISO 的明显 北传特征, 湿位相和干位相的间隔不清楚, 与观测结 果相差很大.

关于北半球夏季 $\operatorname{CISO}$ 在 $\operatorname{SCS}\left(100^{\circ} \sim 120^{\circ} \mathrm{E}\right)$ 平均 的经向传播特征, 观测(图 1(c))中能清楚看见五月中 旬爆发的湿位相及其相对应的干位相阶段性地向北 推进, 八月后 CISO 信号迅速减小. 但是在三种积云 对流参数化方案模拟结果中干湿位相都十分混乱. 相对而言 Manabe 方案(图 1(f))和 Tiedtke 方案(图 1(1)) 的 CISO 信号略好一些, 可以大概看出雨带随时间向 北推进, 但速度偏快且 $10^{\circ} \mathrm{N}$ 以南 CISO 信号几乎消 失.

干湿位相的传播是与季风进退联系的, CISO 的 北传和季风爆发也有着一定的关联.

图 2 给出了印度南部地区 $\left(7.5^{\circ} \sim 12.5^{\circ} \mathrm{N}\right.$, $\left.75^{\circ} \sim 80^{\circ} \mathrm{E}\right) 1980 \sim 2006$ 年平均的印度夏季风爆发前后 OLR 异常和模拟降水量异常的逐日演变过程. 印度 夏季风爆发日期是根据南阿拉伯海 $\left(5^{\circ} \sim 15^{\circ} \mathrm{N}\right.$, $\left.40^{\circ} \sim 80^{\circ} \mathrm{E}\right)$ 区域平均的持续强的 $850 \mathrm{hPa}$ 纬向风( $>6.2$ $\mathrm{m} \mathrm{s}^{-1}$ 超过 $6 \mathrm{~d}$ ) 的开始时间确定的 ${ }^{[33]}$. 由图 2 可知, 观 测中, 在印度夏季风爆发(6 月 3 日)后, 气候态 OLR 异常有一明显的下降过程, 意味着印度南部地区进 入雨季, 同时 CISO 发展至最大湿位相. 在三种积云 对流参数化方案的模拟结果中, 季风爆发日期均偏 早, 只有 Tiedtke 方案模拟的日降水量更接近观测, 并且它更能体现出季风爆发后雨量迅速增加的特征.

综合上面的分析可见，三个区域中, BOB 区域的 CISO 模拟结果与观测更接近. 结合图 1 和 2 的结果, Tiedtke 方案模拟 CISO 结果虽然有很多与观测不符 的地方, 但是却是三种积云对流参数化方案中的最 优选择.

\section{SAMIL2.2.3 模式对 TISO 的模拟}

运用平均功率谱分析方法对 OLR 观测资料的分
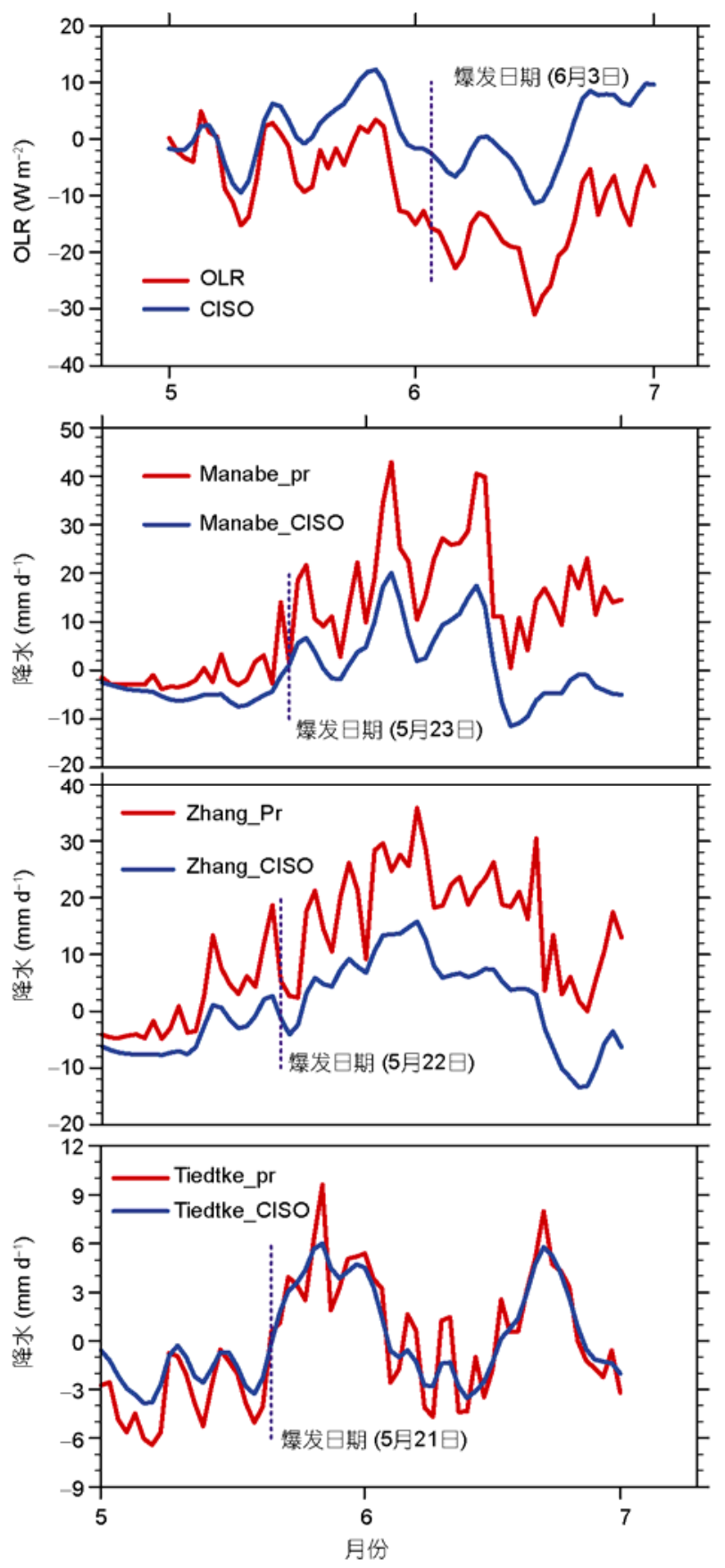

图 2 印度夏季风爆发前后的演变过程

通过 1980 2006年气候态平均的 OLR(NOAA) 异常和三种积云对流参 数化方案的模拟降水量异常得到在印度南部地区 $\left(7.5^{\circ} \sim 12.5^{\circ} \mathrm{N}, 75^{\circ} \sim\right.$ $80^{\circ} \mathrm{E}$ ), 印度夏季风爆发前后的演变过程. 虚线表示气候态情况下的 爆发日期. 红实线为 OLR 或模拟降水量异常, 蓝实线为分别用 OLR 或模拟降水量得出的 CISO. 单位: $\mathrm{W} \mathrm{m}^{-2}$ (OLR), $\mathrm{mm} \mathrm{d}^{-1}$ (降水)

析可得出 BOB 区域, IDO 区域和 SCS 区域的两个主 要的 TISO 模态(12 25d 和 27 50 d). 下面对 12 25 d 
和 27 50 d 的 TISO 模态分析其纬向传播特征. 分别 取计算参考区域为 BOB, IDO 和 SCS 区域, 运用单点 超前滞后相关方法得到一系列的时间-经度剖面图 (图 3 5).
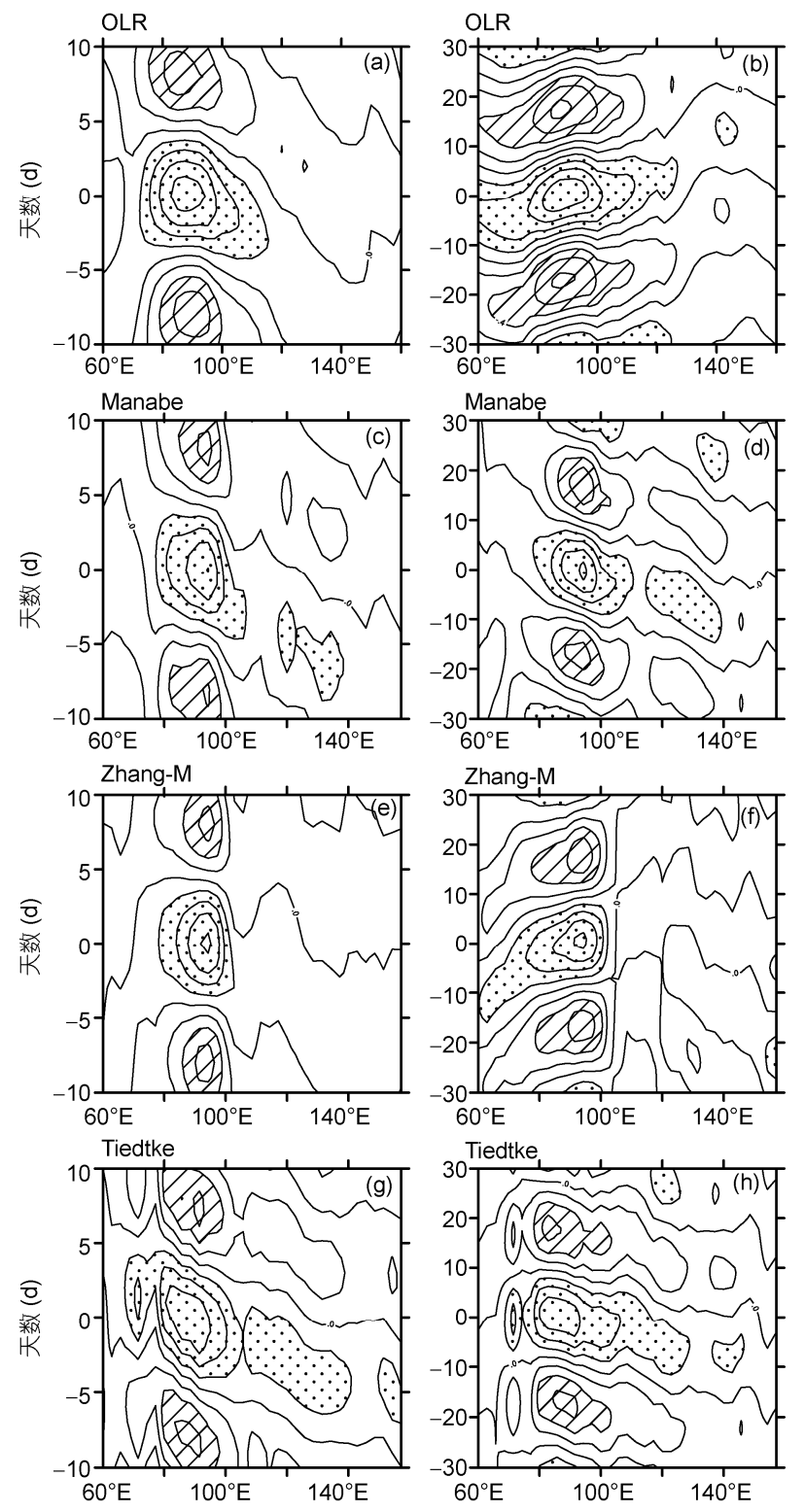

图 3 北半球夏季 $10^{\circ} \sim 20^{\circ} \mathrm{N}$ 平均的两种 TISO 模态的纬向 传播特征

(a), (c), (e) 和 (g) 表示的是利用 OLR 资料和三种积云参数化方案模拟 的 SAMIL2.2.3 日降水资料计算的 12 25 天 TISO 模态的纬向传播; (b), (d), (f) 和(h) 表示的是利用 OLR 资料和三种积云参数化方案模拟 的 SAMIL2.2.3 日降水资料计算的 27 50 天 TISO 模态的纬向传播. 计算方法为单点超前滞后相关. 纵坐标为超前滞后天数. 计算参考 区域为 BOB 区域 $\left(10^{\circ} \sim 20^{\circ} \mathrm{N}, 80^{\circ} \sim 100^{\circ} \mathrm{E}\right)$. 阴影区通过 $99 \%$ 信度检验
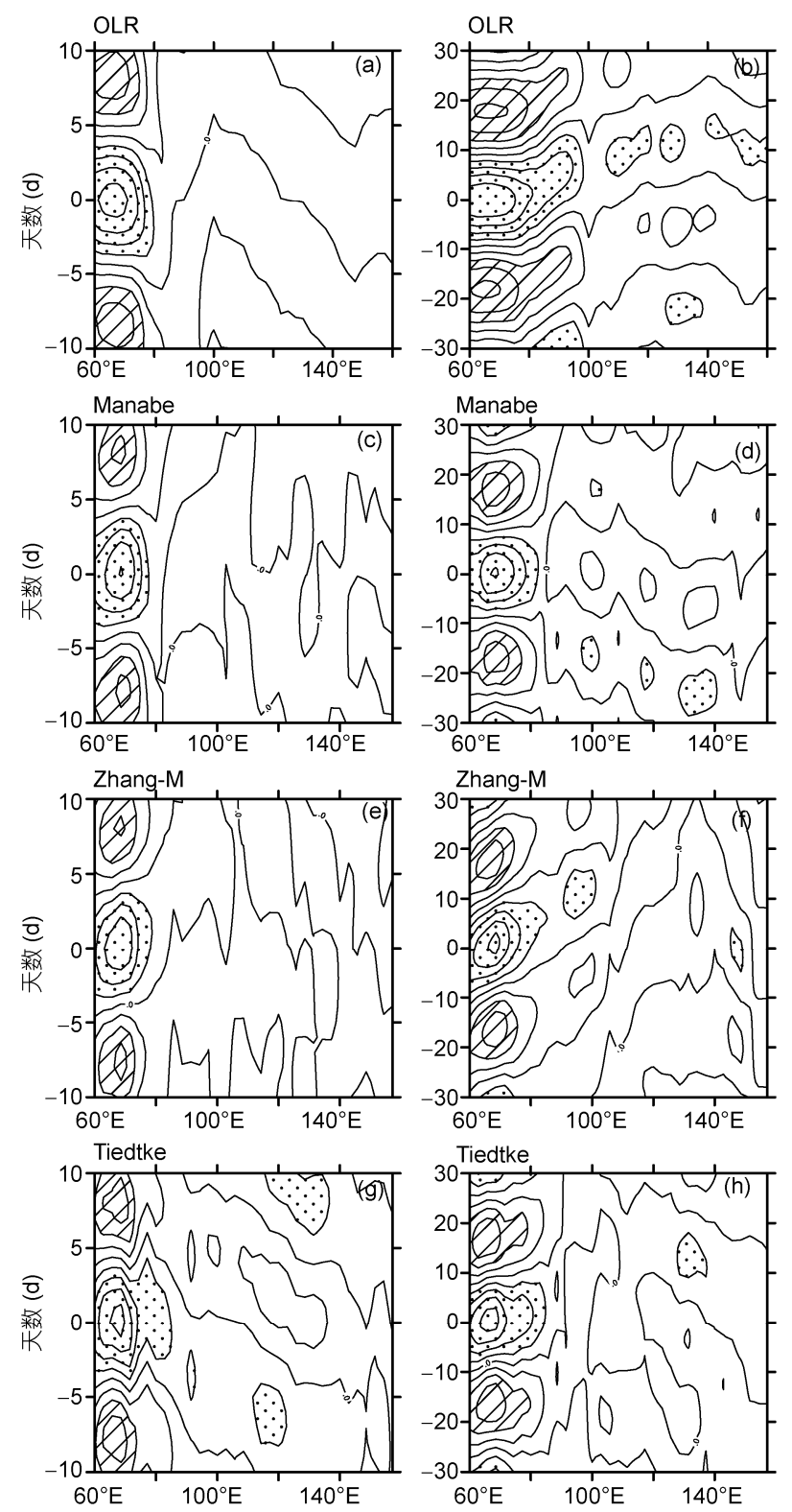

图 4 北半球夏季 $10^{\circ} \sim 20^{\circ} \mathrm{N}$ 平均的两种 TISO 模态的纬向 传播特征

与图 3 类似, 只是计算参考区域取为 $\operatorname{IDO}\left(10^{\circ} \sim 20^{\circ} \mathrm{N}, 50^{\circ} \sim 75^{\circ} \mathrm{E}\right)$

由图 3 5 可看出, 观测表明(1) 12 25 天 TISO 模 态在 $60^{\circ} \sim 70^{\circ} \mathrm{E}$ 的经度范围内具有东传特征, $100^{\circ} \mathrm{E}$ 以 东表现为明显西传; (2) 在 $70^{\circ} \sim 120^{\circ} \mathrm{E}$ 之间, 27 50 天 $\mathrm{TISO}$ 模态向东传播, $120^{\circ} \mathrm{E}$ 以东转为西传. 模拟结果 显示，当参考区域选在 $\mathrm{BOB}$ 时(图 3), ZhangMcFarlane 方案能很好的模拟两种 TISO 模态在 $60^{\circ} \sim 90^{\circ} \mathrm{E}$ 的向东传播特征, Tiedtke 方案可以模拟出 

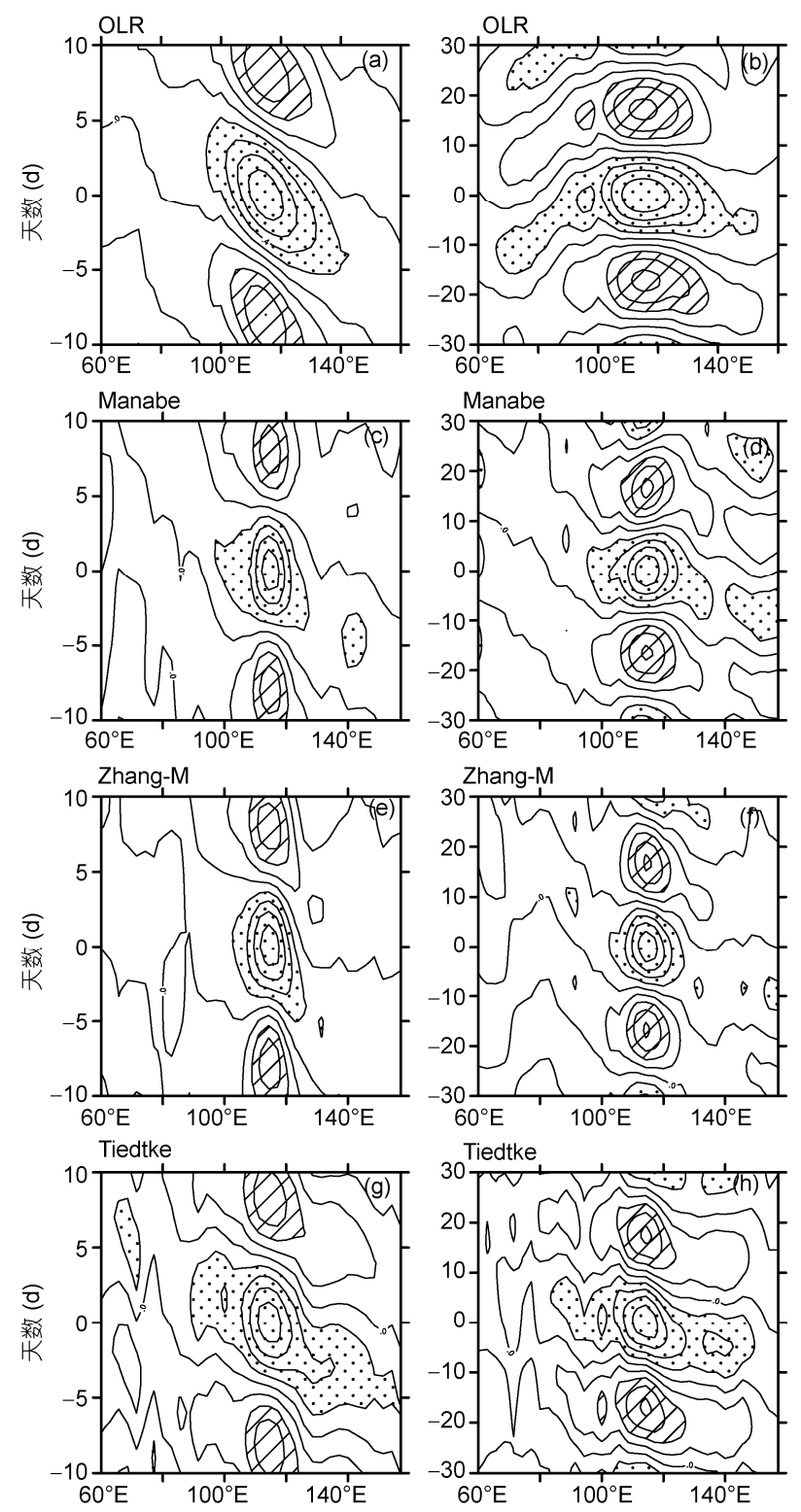

图 5 北半球夏季 $10^{\circ} \sim 20^{\circ} \mathrm{N}$ 平均的两种 TISO 模态的纬向 传播特征

与图 3 类似, 只是计算参考区域取为 $\operatorname{SCS}\left(10^{\circ} \sim 20^{\circ} \mathrm{N}, 110^{\circ} \sim 120^{\circ} \mathrm{E}\right)$

27 50 天 TISO 模态在 $60^{\circ} \sim 75^{\circ} \mathrm{E}$ 之间的东传信号, Manabe 方案却无法模拟出两种 TISO 模态的东传特 征; 当参考区域选为 IDO 时(图 4), 三种积云参数化 方案都能模拟出两种 TISO 模态在印度洋经度范围内 的一些东传特征, 但相较之下 Zhang-McFarlane 方案 模拟两种 TISO 模态东传特征的结果与观测更接近; 当参考区域选为 SCS 时(图 5), 只有 Zhang-McFarlane 方案可以模拟出 $27 \sim 50$ 天 TISO 模态在 $60^{\circ} \sim 80^{\circ} \mathrm{E}$ 经
度范围内的东传特征. 由此可知, Zhang-McFarlane 方 案在模拟 TISO 模态东传这一方面较于其他两种积云 参数化方案具有更大的优越性.

SAMIL2.2.3 版本使用的是新修改的 ZhangMcFarlane 方案, 而在之前的版本中使用的旧 ZhangMcFarlane 方案是无法模拟出 TISO 模态的东传特征 的. 可见, 采用新 Zhang-McFarlane 方案是更有利于 模拟 27 50 天 TISO 模态东传.

\section{4 非绝热加热垂直分布与深浅对流相互作 用}

模式模拟的 ISO 在某些程度上和垂直加热结构 有关, 而模式中的大气加热过程又在很大程度上由 积云对流参数化方案决定.

先给出观测、SAMIL2.2.3 三种积云参数化方案 和旧 Zhang-McFarlane 方案模拟出的非绝热加热垂直 廓线图(见图 6). 在三个区域中, SAMIL2.2.3 模式中 的三种积云参数化方案非绝热加热廓线在对流层中 层都存在最大值. 其中 Tiedtke 方案的非绝热加热最 大加热强度相比于其他廓线更大，在 SCS 区域中可 达到 $8 \mathrm{~K} \mathrm{~d}^{-1}$. 以 BOB 区域为例, 旧 Zhang- McFarlane 方案所模拟的加热强度比新方案要小, 并且在 900 , 600 和 $200 \mathrm{hPa}$ 各有一个加热中心. 而新 ZhangMcFarlane 方案的加热强度与观测接近, 在 900 和 500 $\mathrm{hPa}$ 各有一加热中心.

李崇银等 ${ }^{[34]}$ 通过修改 SAMIL_R42L9 中 Manabe 方案的非绝热加热廓线来说明非绝热加热若在对流 层低层达到最大, 会产生具有东传特征的行星尺度 季节内扰动, 其位相速度与 $\mathrm{MJO}$ 相似.

为进一步说明新 Zhang-McFarlane 方案的不同非 绝热加热廓线垂直分布对模拟 27 50 天 TISO 模态东 传的影响, 对该方案进行三个敏感性试验 (CUP, CDN 和 CLHO).

前两个敏感性试验(CUP 和 CDN)的目的是通过 改变模式产生的非绝热加热的垂直分布, 分析模式 模拟的 27 50 天 TISO 模态对其的敏感性. CUP 具体 的试验设计如下: 首先将热带地区 $\left(20^{\circ} \mathrm{S} 20^{\circ} \mathrm{N}\right)$ 大气 非绝热加热廓线修改为最大加热位于对流层上层的 加热廓线. 由于第 11 层和第 12 层所在的高度大约在 250 300 hPa, 位于对流层上层, 所以我们保留这两 层的加热暂时不做修改, 将其他层的加热减小到原 

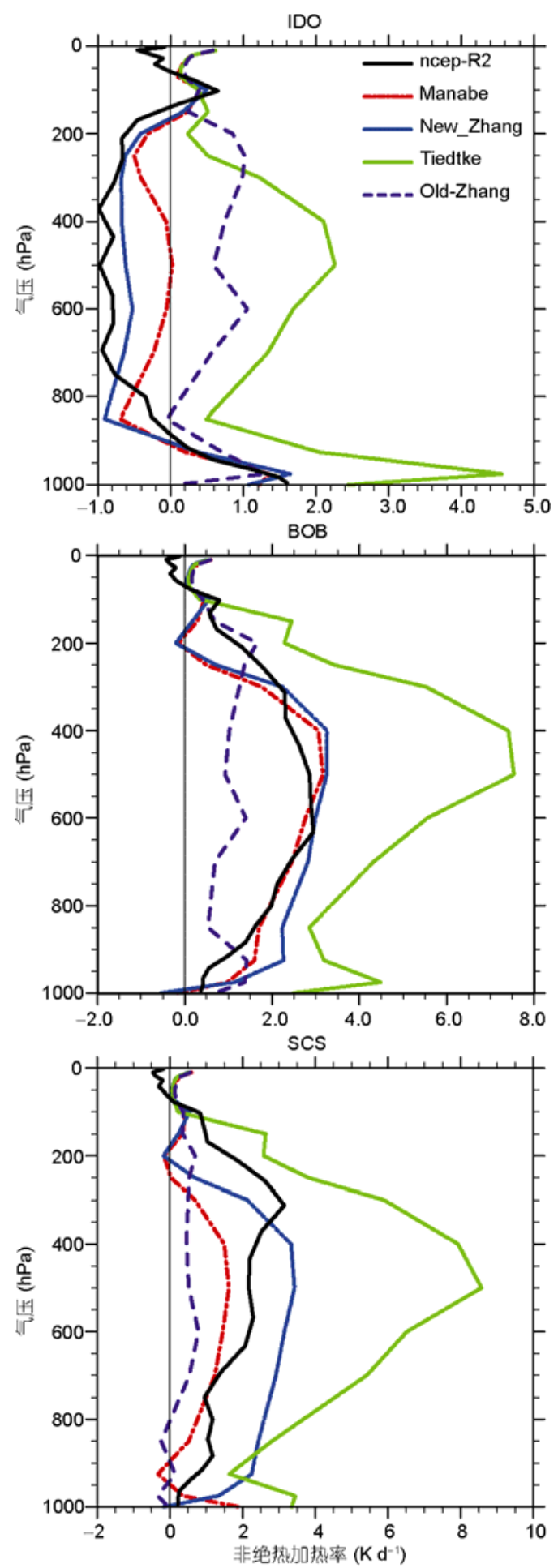

图 6 北半球夏季 $\mathrm{BOB}, \mathrm{IDO}$ 和 SCS 地区平均的非绝热加 热垂直廓线

所用资料分别为 NcepR2 再分析资料和由 SAMIL2.2.3 模式中三种 积云参数化方案模拟所得数据, 以及 SAMIL2.08 模式中 Zhang-McFarlane 方案模拟所得数据
来的 $10 \%$. 为了保持能量守恒，需要将减少的加热总 量均匀地再加到第 11 和第 12 层上, 所以最后得到第 11 层和第 12 层修改之后的大气非绝热加热值. 再在 南北纬 $20^{\circ} \sim 30^{\circ}$ 之间逐渐减少每层的大气非绝热加热 的改变量(由 $90 \%$ 逐渐变到 0 ), 而在南北纬 $30^{\circ}$ 以外就 不再改变大气非绝热加热廓线; 使用相同的方法可 以在 $\mathrm{CDN}$ 试验中使得大气非绝热加热最大值位于低 层，在该试验中我们选择第 6 和第 7 层(约为 600 700 $\mathrm{hPa}$ ). 在模式中每次调用积云对流参数化过程时改变 大气非绝热加热廓线, 然后再将改变了的加热廓线 放入模式之中, 进行下一步计算. 第三个敏感性试验 (CLH0)的目的是进一步确定潜热加热在模拟 27 50 天 TISO 模态中的作用. 具体做法是将潜热加热取消, 只剩下感热加热和辐射的作用. 图 7 是三个敏感性试 验对应的非绝热加热垂直廓线，代表了三种不同的 垂直加热分布.

图 8 是三个试验模拟的 27 50 天 TISO 模态的纬 向传播特征. 无论是 CUP 还是 CDN, 都可以模拟出 $80^{\circ} \sim 100^{\circ} \mathrm{E}$ 的东传特征. 结果表明, 在新 ZhangMcFarlane 方案中, 修改加热中心位于高层或低层, 对 27 50 天 TISO 模态的东传影响不大. 但是如果取 消潜热加热的作用(CLH0)，基本不能模拟出 27 50 天 TISO 模态的东传, 可见潜热加热对 TISO 东传模 拟是起决定性作用的.

在模式 SAMIL2.2.3 中, 深对流参数化方案选用

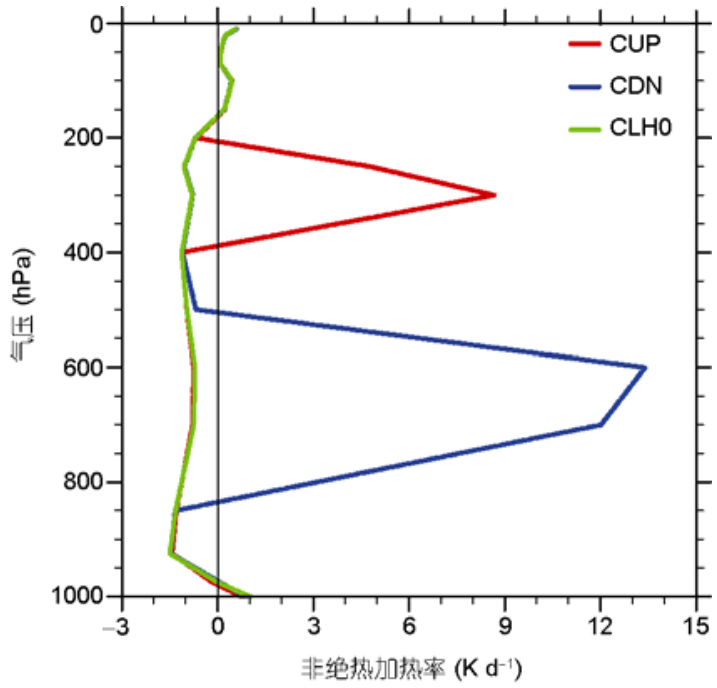

图 $7 \mathrm{CUP}, \mathrm{CDN}$ 和 CLHO 试验对应的北半球夏季 $\left(10^{\circ} \sim 20^{\circ} \mathrm{N}, 5^{\circ} \sim 120^{\circ} \mathrm{E}\right)$ 区域平均的非绝热加热垂直廓线 

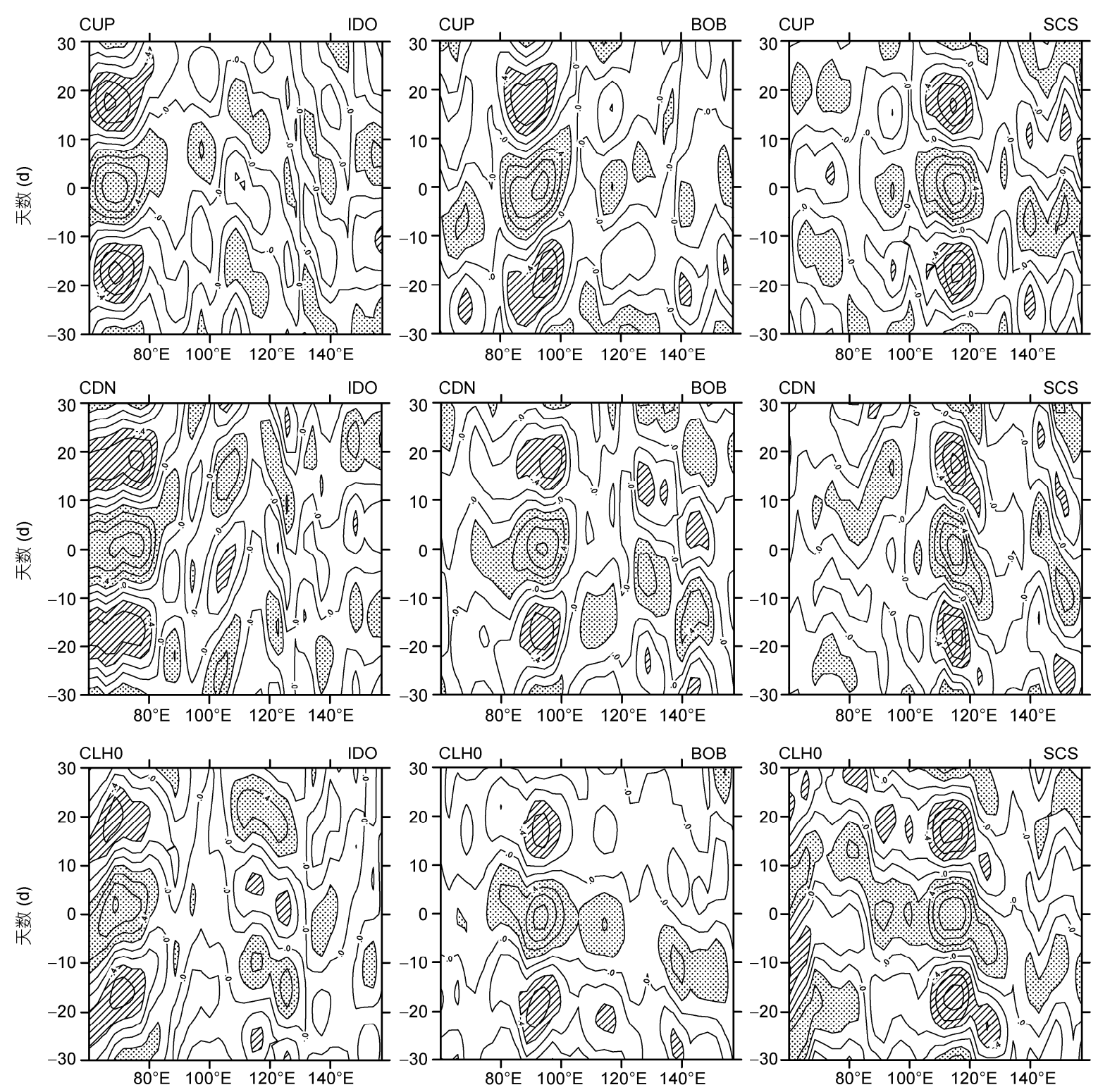

图 $8 \mathrm{CUP}, \mathrm{CDN}$ 和 CLHO 试验对应的北半球夏季 $10^{\circ} \sim 20^{\circ} \mathrm{N}$ 平均的 $27 \sim 50$ 天 TISO 模态纬向传播特征

所用资料是试验所得的日降水量. 计算方法为单点超前滞后相关. 计算参考区域为分别为 IDO, BOB 和 SCS 区域. 阴影区通过 $99 \%$ 信度检验

Zhang-McFarlane 方案时, 浅对流方案相应地取为 Hack 方案. 设计一个敏感性试验(NS), 取消浅对流 的作用, 即不进行 Hack 方案的计算, 其目的是说明 深浅对流相互作用对模拟 27 50 天 TISO 模态东传的 影响. 试验结果表明当去掉浅对流之后, 深对流也受 到抑制, SAMIL2.2.3 模拟不出 27 50 天 TISO 模态东 传特征(图略). 以 $\mathrm{BOB}$ 为例, 试验产生的非绝热加热 廓线如图 9 所示. 新 Zhang-McFarlane 方案在忽略浅 对流作用之后的加热垂直分布与旧 Zhang-McFarlane
方案十分一致, 并且都模拟不出东传特征. 从中可以 看出, 在 Zhang-McFarlane 方案中, 浅对流对于深对 流的产生起着关键的作用，深浅对流相互作用对模 拟 TISO 模态东传也是至关重要的.

综上所述可知: (1) 新旧 Zhang-McFarlane 方案 非绝热加热廓线, 加热强度与加热中心位置都很不 同. 旧 Zhang-McFarlane 方案与去掉浅对流之后的新 Zhang-McFarlane 方案加热强度大小与加热中心位置 十分相似, 并且两者都不能模拟出 27 50 天 TISO 模 


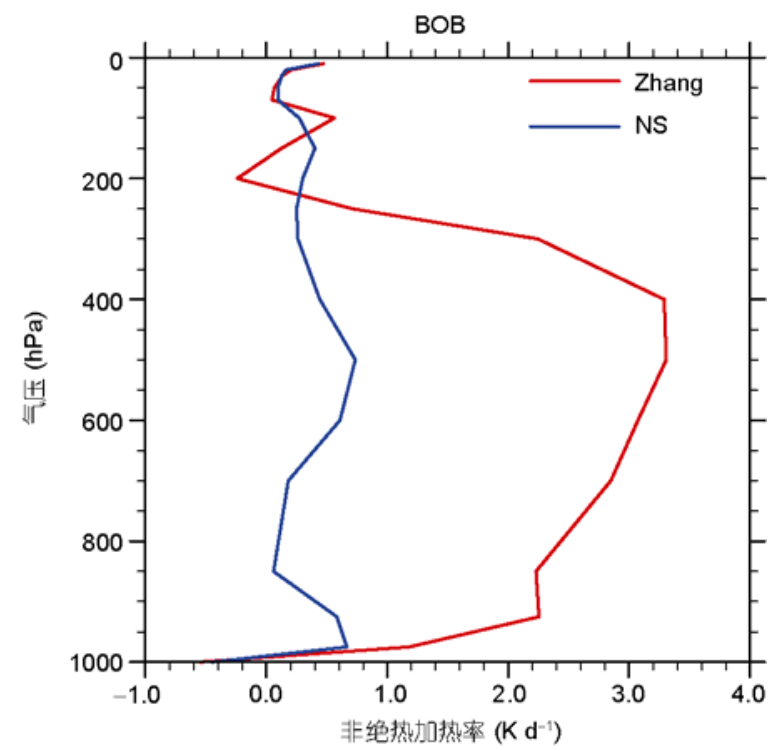

图 9 北半球夏季 BOB 地区平均的非绝热加热垂直廓线 红线为新 Zhang-McFarlane 方案, 蓝线为 NS 试验

态东传. 这说明模拟 27 50 天 TISO 模态东传与加热 的垂直分布和加热强度有着比较紧密的关系. (2) 新 Zhang-McFarlane 方案中, 模拟 27 50天 TISO 模态东 传对于加热中心所在位置不是很敏感. (3) 潜热加热 在模拟 27 50天 TISO 模态起着决定性的作用. (4) 新 Zhang-McFarlane 方案中, 取消浅对流作用, 深对流 同样被抑制, 模拟不出 27 50 天 TISO 模态东传. 可 见深浅对流相互作用对其东传的模拟起着关键作用.

\section{SAMIL2.2.3 与 SAMIL2.08 两种版本 Manabe 方案模拟结果的比较}

SAMIL2.2.3 模式中 Manabe 方案本身并没有进 行改进, 只是针对某些问题改变了物理过程其他部 分的辐射方案和气溶胶条件. SAMIL2.08 模式选用 Manabe 方案可以模拟出 TISO 模态的东传特征, 但 SAMIL2.2.3 版本的 Manabe 方案却无法模拟(如图 10, 11(a) (d)). 为了进一步弄清导致无法模拟 TISO 模态 东传的原因, 做了一项敏感性试验 (M_na), 即在 SAMIL2.2.3 中不考虑气溶胶的直接效应, 再与 SAMIL2.08 作比较.

当参考区域为 BOB 区域时(如图 10), SAMIL2.08 中的 Manabe 方案可以模拟出两种 TISO 模态在 $60^{\circ} \sim 90^{\circ} \mathrm{E}$ 的向东传播, 与观测一致; 而 SAMIL2.2.3
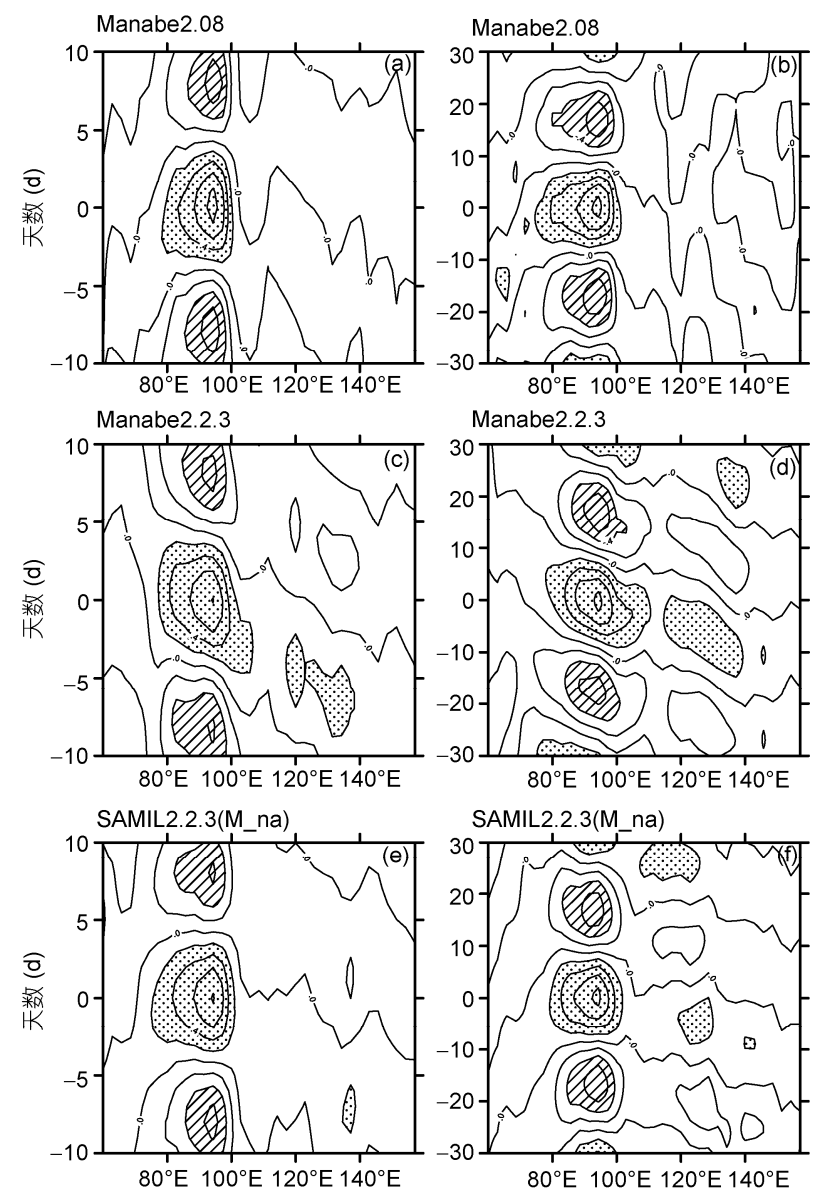

SAMIL2.2.3(M_na)

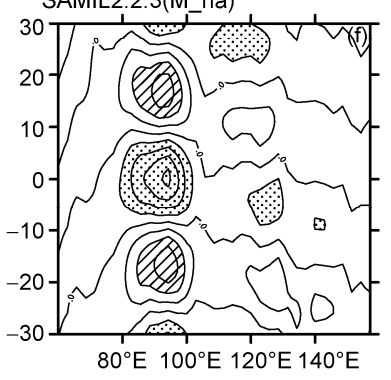

图 $1010^{\circ} \sim 20^{\circ} \mathrm{N}$ 平均的两种 TISO 模态的纬向传播特征 (a)和(c)表示的是利用 SAMIL2.08 版本和 SAMIL2.2.3 版本 Manabe 积云参数化方案模拟的日降水资料计算的 12 25 天 TISO 模态的纬 向传播特征; (b)和(d)表示的是利用 SAMIL2.08 版本和 SAMIL2.2.3 版本 Manabe 积云参数化方案模拟的日降水资料计算的 27 50 天 TISO 模态的纬向传播特征. (e)和(f) 分别表示的是 NA 敏感性试验所 得日降水资料计算的 12 25 天 TISO 模态和 27 50 天 TISO 模态的 纬向传播特征. 计算方法为单点超前滞后相关. 计算参考区域为 BOB 区域. 阴影区通过 99\%信度检验

中 Manabe 方案模拟两种 TISO 模态结果在 $60^{\circ} \sim 160^{\circ} \mathrm{E}$ 均表现为西传. 而参考区域为 IDO 区域时(如图 11), 虽然两个版本的 Manabe 方案都可以模拟出东传, 但 是 SAMIL2.08 中 Manabe 方案模拟的 TISO 分量与观 测更接近. 当参考区域为 SCS 区域(与 BOB 区域结果 类似，图略), SAMIL2.2.3 中 Manabe 方案模拟出的 TISO 模态均表现为西传, SAMIL2.08 中 Manabe 方案 模拟结果与观测类似，其模拟的 TISO 分量具有东传 特征. M_na 试验中所反映的 TISO 模态纬向传播特征 与 SAMIL2.08 非常接近, 可以模拟出其东传. 

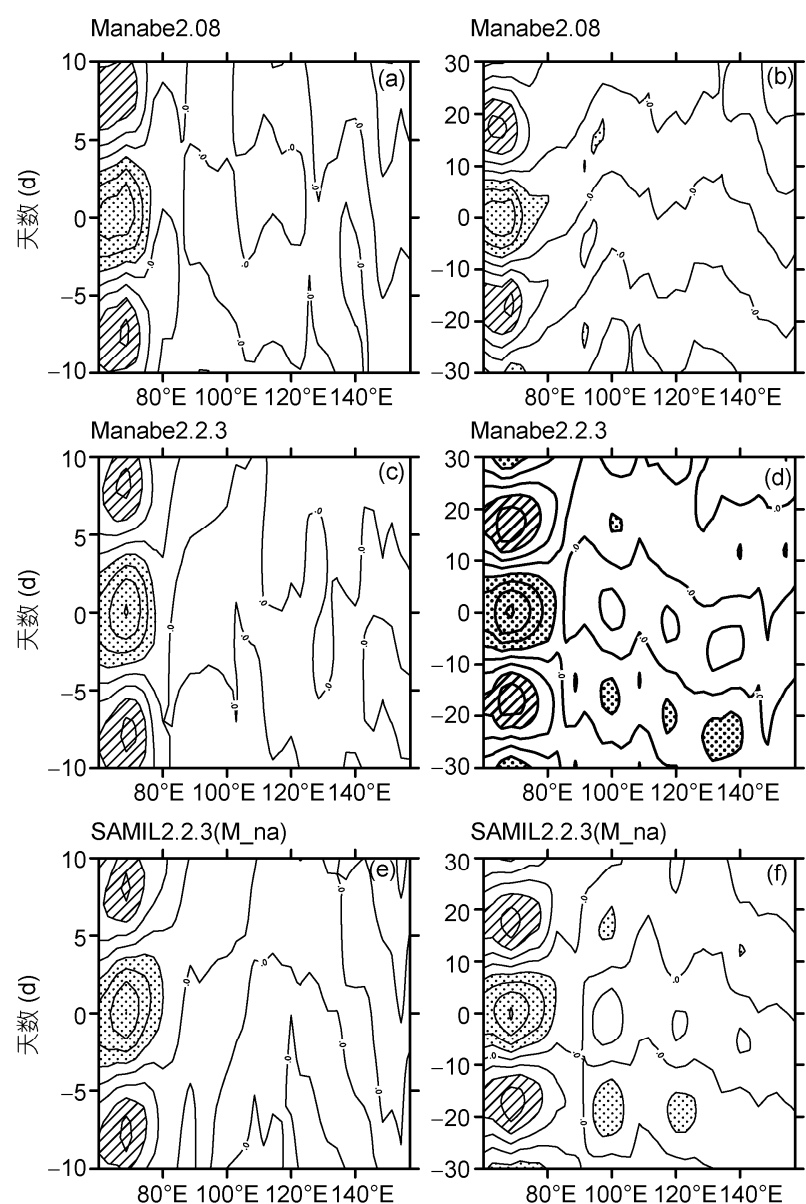

$80^{\circ} \mathrm{E} 100^{\circ} \mathrm{E} 120^{\circ} \mathrm{E} 140^{\circ} \mathrm{E}$
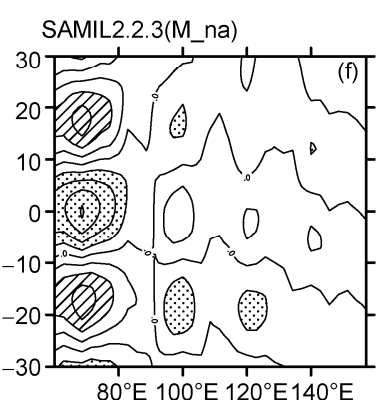

图 $111^{\circ} \sim 20^{\circ} \mathrm{N}$ 平均的两种 TISO 模态的纬向传播特征

与图 10 类似，只是计算参考区域取为 IDO 区域

由此可见, 虽然 SAMIL2.2.3 没有改变 Manabe 方案, 但是模式中新增加的气溶胶直接效应仍然影 响到同一积云对流参数化方案对 TISO 的模拟结果.

\section{6 结论和讨论}

本文应用中国科学院大气物理研究所 LASG 的 大气环流谱模式(SAMIL2.2.3)研究了不同积云对流 参数化方案造成的热带大气低频振荡模拟结果的显
著差异, 并且通过比较模式两个版本 (SAMIL2.08 和 SAMIL2.2.3)的同一积云对流参数化方案对 ISO 模拟 结果的差异说明其他物理过程的改变也会导致 ISO 模拟结果的明显不同. 本文主要结论如下:

(1) 亚洲热带季风区气候态季节内振荡(CISO) 存在明显的阶段性北传特征. 比较三种积云参数化 方案 CISO 模拟，结果表明 Tiedtke 方案在三个地区的 模拟结果相对于其他两个方案更接近观测.

（2）亚洲热带季风区内季节内振荡的年际变化 部分(TISO)的不同模态纬向传播特征存在差异, 其 中 27 50 天分量具有明显的东传特征. 新 ZhangMcFarlane 方案在经过改进之后, 其模拟 TISO 东传 的能力也得到进一步加强.

(3) 模拟 27 50 天 TISO 模态东传与加热的垂直 分布和加热强度有着比较紧密的关系; 新 Zhang-McFarlane 方案中, 模拟 27 50 天 TISO 模态东 传对于加热中心所在位置不敏感; 潜热加热在模拟 27 50 天 TISO 模态东传起着决定性的作用; 新 Zhang-McFarlane 方案中, 取消浅对流作用, 深对流 同样被抑制, 模拟不出 27 50 天 TISO 模态东传. 可 见深浅对流相互作用对其东传的模拟至关重要.

(4) SAMIL2.2.3 和 SAMIL2.08 中 Manabe 积云对 流参数化方案完全相同. 但只有后者能很好地模拟 出 27 50 天 TISO 模态的东传. 这说明模式中新增加 的气溶胶直接效应仍然影响到同一积云对流参数化 方案对 TISO 的模拟结果. 因此, 模式发展过程中有 针对性的物理参数化方案的改进并非在每一方面上 都产生正面效果. 动力框架和物理过程, 以及不同物 理过程之间的协调发展是改进模式的重要途径.

本文通过一系列敏感性试验，对影响模拟 27 50 天 TISO 模态东传的因素有了一定的认识, 但是对于 模拟 CISO 北传的影响因子并没进行深入研究. 已有 的很多工作都表明, 海气耦合模式比大气环流模式 更能准确抓住 ISO 北传特征, 并且海气相互作用有可 能是热带大气季节内振荡产生的原因之一 ${ }^{[35]}$. 关于 海气耦合对 ISO 模拟的影响, 我们将另文讨论. 
1 Madden R A, Julian P R. Detection of a 40-50 day oscillation in the zonal wind in the tropical Pacific. J Atmos Sci, 1971, 28: 702-708

2 Madden R A, Julian P R. Description of global-scale circulation cells in the tropics with a 40-50 day period. J Atmos Sci, 1972, 29: 1109-1123

3 Madden R A, Julian P R. Observations of the 40-50 day tropical oscillation-A review. Mon Weather Rev, 1994, 122: 814-837

4 Lorenc A C. The evolution of planetary scale $200 \mathrm{mb}$ divergence flow during the FGGE year. Q J R Meteorol Soc, 1984, 110: 427-441

5 Weickmann K M, Lussky G R, Kutzbach J E. Intraseasonal (30-60 day) fluctuations of outgoing longwave radiation and 250 mb streamfunction during Northern winter. Mon Weather Rev, 1985, 113: 941-961

6 Murakami T, Chen L X, Xie A, et al. Eastward propagation of 30-60 day perturbations as revealed from outgoing long-wave radiation data. J Atmos Sci, 1986, 43: 961-971

7 Krishnamurti T N, Bhalme H N. Oscillation of a monsoon system, Part I: Observational aspects. J Atmos Sci, 1976, 33: 1937-1954

8 李崇银, 周亚萍. 热带大气中的准双周(10-20 天)振荡. 大气科学, 1995, 19: 435-444

9 Yasunari T. A quasi-stationary appearance of 30 to 40 day period in the cloudiness fluctuations during the summer monsoon over India. $\mathbf{J}$ Meteorol Soc Jpn, 1980, 58: 225-229

10 Liebmann B, Hendon H H, Glick J D. The relationship between tropical cyclones of the western Pacific and Indian Oceans and the Madden-Julian Oscillation. J Meteorol Soc Jpn, 1994, 72: 401-411

11 李崇银, 周亚萍. 热带大气季节内振荡和 ENSO 的相互关系. 地球物理学报, 1994, 37: 17-26

12 李桂龙, 李崇银. 大气季节内振荡的活动与 El Nino. 热带气象学报, 1998, 14: 54-62

13 Hendon H H, Liebmann B, Newman M, et al. Mediumrange forecasts errors associated with active episodes of the Madden-Julian Oscillation. Mon Weather Rev, 2000, 128: 69-85

14 Slingo J M, Sperber K R, Boyle J S, et al. Intraseasonal oscillation in 15 atmospheric general circulation models: Results from an AMIP diagnostic subproject. Clim Dyn, 1996, 12: 325-357

15 贾小龙, 李崇银. 热带大气季节内振荡数值模拟对积云对流参数化方案的敏感性. 气象学报. 2007, 65: 837-855

16 Chao W C, Deng L. Tropical intraseasonal oscillation, supper cloud clusters, and cumulus convection schemes. Part II: 3D aquaplanet simulations. J Atmos Sci, 1998, 55: 690-709

17 Wang W, Schlesinger M E. The dependence on convection parameterization of the tropical intraseasonal oscillation simulated in the UIUC 11-layer atmospheric GCM. J Clim, 1999, 12: 1423-1457

18 Maloney E D, Hartmann D L. The sensitive of intraseasonal variability in the NCAR CCM3 to changes in convection parameterization. J Clim, 2001, 14: 2015-2034

19 Wang B, Xu X H. Northern hemisphere summer monsoon singularities and climatological intraseasonal oscillation. J Clim, 1997, 10: 1071-1085

20 杨静. 东亚和西北太平洋夏季降水中“气候态”与“非气候态”的季节内振荡. 博士学位论文. 北京：中国科学院大气物理研究所, 2008 . $1-15$

21 Liebmann B, Smith C A. Description of a complete (interpolated) outgoing longwave radiation dataset. Bull Amer Meteorol Soc, 1996, 77: 1275-1277

22 Kanamitsu M, Ebisuzaki W, Woollen J. NCEP/DOE AMIP-II Reanalysis (R-2). Bull Amer Meteorol Soc, 2002, 83: 1631-1643

23 曾庆存. 大气运动的特征参数和动力学方程. 气象学报, 1963, 33: 472-483

24 Edwards J M, Slingo A A. Studies with a flexible new radiation code. I: Choosing a configuration for a large-scale model. Q J R Meteorol Soc, 1996, 122: 689-720

25 Xue Y, Sellers P J, Kinter J J, et al. A simplified biosphere model for global climate studies. J Clim, 1991, 4: 345- 364

26 Liu H, Wu G X. Impacts of land surface on climate of July and onset of summer monsoon: A study with an AGCM plus SSib. Adv Atmos Sci, 1997, 15: 410-423

27 周天军, 王在志, 宇如聪, 等. 基于 LASG/IAP 大气环流谱模式的气候系统模式. 气象学报, 2005, 63: 702-715

28 Manabe S, Smagorinsky J, Strickler R F. Simulated climatology of general circulation model with a hydrological cycle. Mon Weather Rev, 1965, 93: 769-798

29 Zhang G J. Convective quasi-equilibrium in midlatitude continental environment and its effect on convective parameterization. J Geophys Res, 2002, 107, doi: 10.1029/2001JD001005

30 Zhang G J, Mu M Q. Simulation of the Madden-Julian Oscillation in the NCAR CCM3 using a revised Zhang-McFarlane convection parameterization Scheme. J Clim, 2005, 18: 4046-4064

31 刘屹岷, 刘琨, 吴国雄. 积云对流参数化方案对大气含水量及降水的影响. 大气科学, 2007, 31: 1201-1211

32 Yasunari T. Cloudiness fluctuations associated with the Northern Hemisphere summer monsoon. J Meteorol Soc Jpn, 1979, 57: 227-242

33 Wang B, Ding Q H, Joseph P V. Objective definition of the Indian summer monsoon onset. J Clim, 2009, 22: 3303-3316

34 Li C Y, Jia X L, Ling J, et al. Sensitivity of MJO simulations to diabatic heating profiles. Clim Dyn, 2009, 32: 167-187

35 杨修群, 黄士松. 海气耦合系统中的季节内振荡模态. 热带气象学报, 1993, 3: 202-210 